\title{
Suppression of Surface-Enhanced Raman Scattering on Gold Nanostructures by Metal Adhesion Layers
}

Loan Le Thi Ngoc, ${ }^{\dagger}$, Tao Yuan, ${ }^{\ddagger}$ Naoto Oonishi, ${ }^{\llbracket}$ Jan van Nieuwkasteele, ${ }^{\S}$ Albert van den Berg, ${ }^{\S}$

Hjalmar Permentier, ${ }^{.}$Rainer Bischoff, ${ }^{,}$and Edwin T. Carlen* ${ }^{\oplus}$

${ }^{\dagger}$ Physics Department, Quy Nhon University, Vietnam

${ }^{\ddagger}$ Analytical Biochemistry Group, University of Groningen, The Netherlands

${ }^{\S}$ BIOS Lab on a Chip Group, University of Twente, The Netherlands

"Graduate School of Pure and Applied Sciences, University of Tsukuba, Japan

*ecarlen@ims.tsukuba.ac.jp; Edwin T. Carlen, University of Tsukuba, 1-1-1 Tennodai, Tsukuba, Ibaraki, Japan 305-8573. Phone: +81298535110.

\section{Supporting information}

1. Au and Ti/Au nanostructures. The nanostructured $\mathrm{Au}$ surfaces are fabricated by depositing (with electron-beam evaporation) thin polycrystalline (PC) $\mathrm{Au}$ and $\mathrm{Ti} / \mathrm{Au}$-bimetal layers on nanopatterned silicon-nitride template surfaces. Figures S1(a) and S1(b) show top and cross section scanning electron microscopy (SEM) views of nanostructured Au surfaces without a Ti adhesion layer $\left(t_{\mathrm{Ti}}=0 \mathrm{~nm}\right)$. Figures $\mathrm{S} 1(\mathrm{c})$ and $\mathrm{S} 1(\mathrm{~d})$ show SEM images of nanostructured PC-Ti/Au surfaces (with Ti adhesion layer, $t_{\mathrm{Ti}}=5 \mathrm{~nm}$ ). These SEM images demonstrate that the Ti adhesion layer does not affect the three-dimensional shape of the Au layer following deposition of the bimetal layer, and therefore, the observed changes of localized surface plasmon resonance characteristics are not the result of shape or geometry changes of the nanostructures.
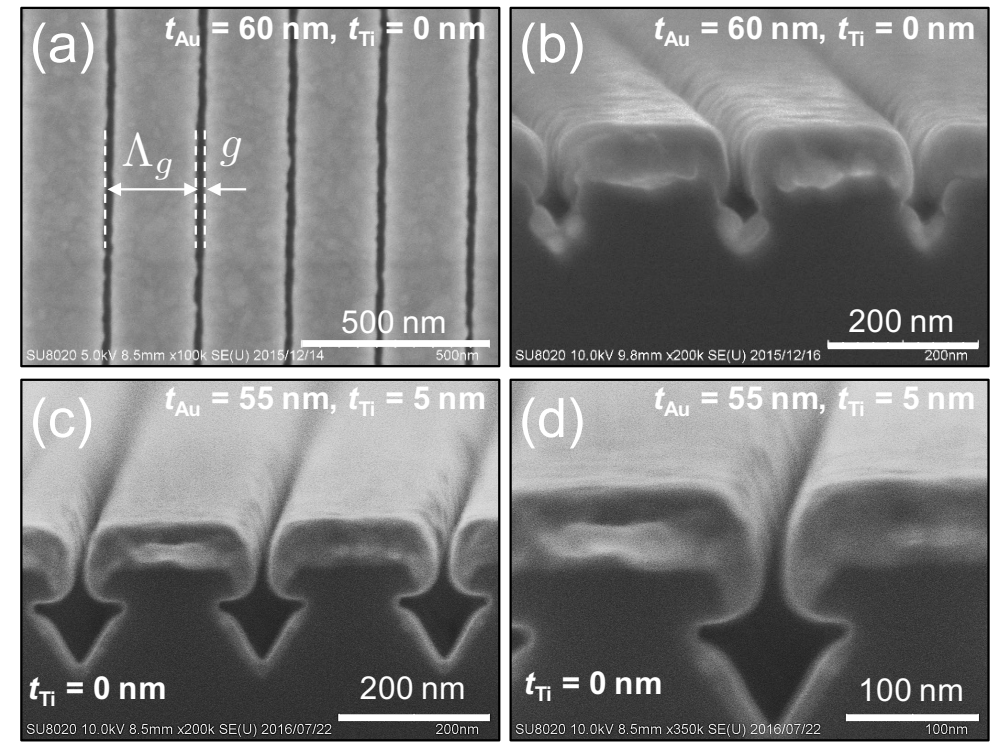

Figure S1. SEMs of nanostructured Au surfaces with pitch $\Lambda_{\mathrm{g}}=250 \mathrm{~nm}$ and nanocavity spacing $g \approx 10 \mathrm{~nm}$. $(\mathrm{a}, \mathrm{b}) 60 \mathrm{~nm}$ Au layer $\left(t_{\mathrm{Au}}=60 \mathrm{~nm}\right)$ and no titanium layer $\left(t_{\mathrm{Ti}}=0 \mathrm{~nm}\right)$. (c, d) $t_{\mathrm{Au}}=55 \mathrm{~nm}$ and $t_{\mathrm{Ti}}=5 \mathrm{~nm}$. 
2. Electric field in the Au layer. The electric field penetrates into the Au layer and the magnitude of the field decays exponentially as a function of $x$ from the interface. Figure S2 shows the calculated (finite difference time domain method) field profile (solid black squares) as a function of $x$ and the fit of an exponential decay model $E_{\mathrm{Au}}(x) / E_{\mathrm{i}}=\mathrm{A}+\operatorname{Bexp}(-x / \delta)$ (red solid line). From the calculated profile the skin depth is $\delta \approx 16 \mathrm{~nm}$.

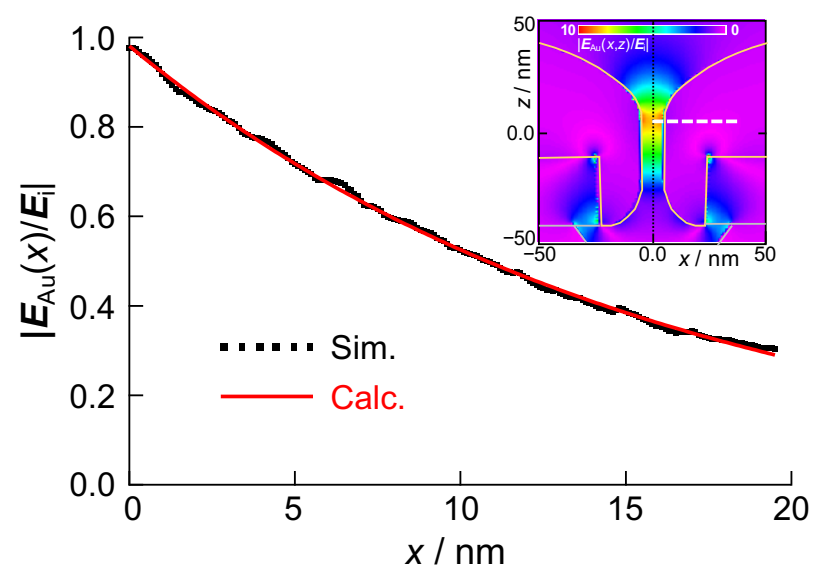

Figure S2. Electric field profile calculated in the Au layer (black solid squares) and corresponding fit (red solid line) to an exponential decay model $E_{\mathrm{Au}}(x) / E_{\mathrm{i}}=\mathrm{A}+\operatorname{Bexp}(-x / \delta)$. The fit model parameters: $\mathrm{A}=-0.158$, $\mathrm{B}=1.383,1 / \delta=0.062$.

3. Impurity interdiffusion. The interdiffusion of metal impurities into the $\mathrm{Au}$ layer during the deposition process (of $\mathrm{Ti} / \mathrm{Au}$ and $\mathrm{Cr} / \mathrm{Au}$ bimetals) has been known for a long time, however, it is not well known with respect to plasmonics and plasmon damping. There is extensive evidence of interdiffusion of Ti and AuTi impurities into PC-Au layers prepared by physical vapor deposition. ${ }^{1,2}$ Both Ti and AuTi impurities have been observed in the grain boundaries of as-deposited Au thin films with diffusivity of $D_{\mathrm{Ti}} \approx 5 \times 10^{-15} \mathrm{~cm}^{2} \mathrm{~s}^{-1}$. This high diffusion rate has been attributed to intense grain boundary diffusion. ${ }^{2}$ Habteyes et al. estimated a diffusion rate of $10 \mathrm{~nm} \mathrm{~min}{ }^{-1}$ for Ti impurities in PC-Au. ${ }^{3}$ In this case, Ti impurities diffuse a distance of approximately $14 \mathrm{~nm}$ from the $\mathrm{Ti} / \mathrm{Au}$ interface, shown in Fig. S3, which would result in an increased interaction volume of Ti with the electric field in the Au layer, compared to the simulated results. This accounts for the large plasmon damping and resonance wavelength redshift observed in the reflectance measurements.

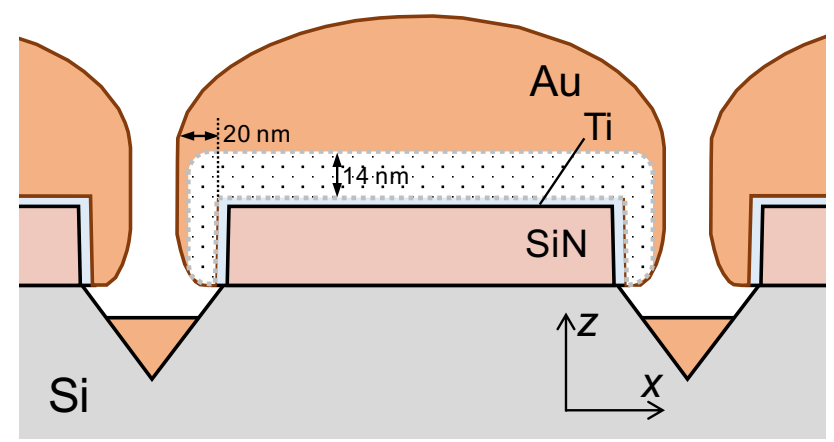

Figure S3. Ti and AuTi interdiffusion profile (white region) in the nanostructure. 
4. SERS of BT Uniformity. A representative SERS spectrum of a benzenethiol (BT) chemisorbed self-assembled monolayer (SAM) on a nanostructured Au surface is shown in Fig. S4 with the dominant Raman active vibrational modes: 416, 691, 994, 1018, 1068, and $1565 \mathrm{~cm}^{-1}$; the 416, 691, and $1068 \mathrm{~cm}^{-1}$ bands are due to ring modes and C-S stretch mode, the $994 \mathrm{~cm}^{-1}$ band is a ring mode, the $1018 \mathrm{~cm}^{-1}$ band from $\mathrm{H}$-stretch, and the $1565 \mathrm{~cm}^{-1}$ band represents a $\mathrm{C}-\mathrm{C}$ ring stretch mode. Note that the absence of the $917 \mathrm{~cm}^{-1}$ vibration mode is an indicator of BT monolayer formation. Each vibration band of a measured SERS spectrum at each measurement location is modeled with a Lorentzian function, and the background signal is modeled with a third order polynomial function. The integrated intensity of each vibration band $\kappa^{\Delta v}$ was calculated by integrating the area of the fitted Lorentzian function after the removal of the background. The fitting accuracy of the Lorentzian function was comparable to the Voigt function. An example of a modeled spectrum is shown in Figure S4(a) (red solid line). Figure S4(b) shows a Raman image of the integrated intensity of the combined ring-bending and C-S stretch modes (1068 $\mathrm{cm}^{-1}$ band) from 2500 full spectra measured over an area of $1600 \mu \mathrm{m}^{2}$, thus demonstrating the high spatial uniformity of the nanostructured PC-Au substrates, where $100 \%$ of the measured locations generate very large Raman intensities. Figure S4(c) shows a histogram of the measured integrated intensity and fitted Gaussian distribution with mean $3.2 \times 10^{4}$ counts $\mathrm{cm}^{-1}$. The measured SERS spectra are highly uniform with every measurement location providing a large enhancement factor with about 9\% variation across the measured surface, which we attribute to the new fabrication technique with controllable and uniform nanogaps with extremely large nanogap length density $\left(1 \mathrm{~km} \mathrm{~cm}^{-2}\right)$ that provides a large number of scattering hot-spots at each measurement location. The sample-tosample variation has been estimated to be about $10 \%$.

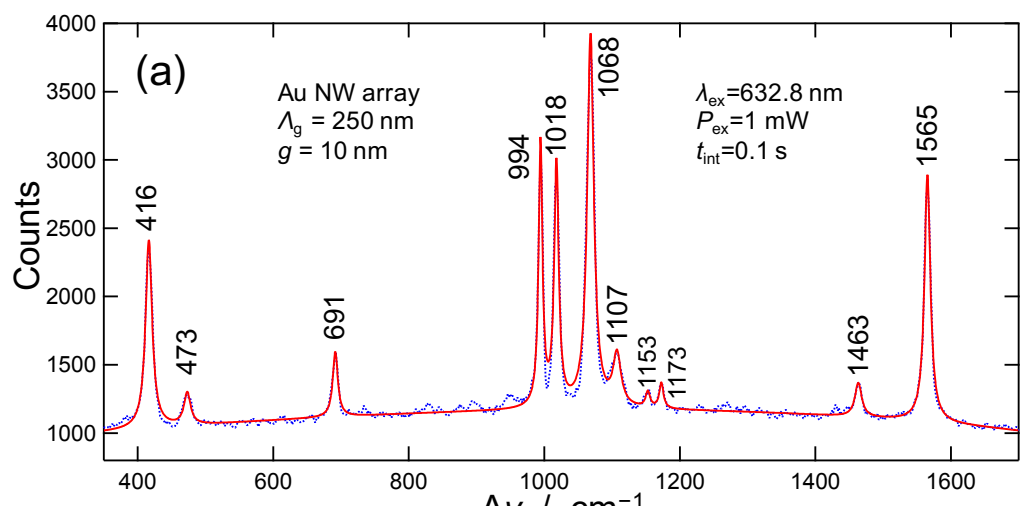

(b)
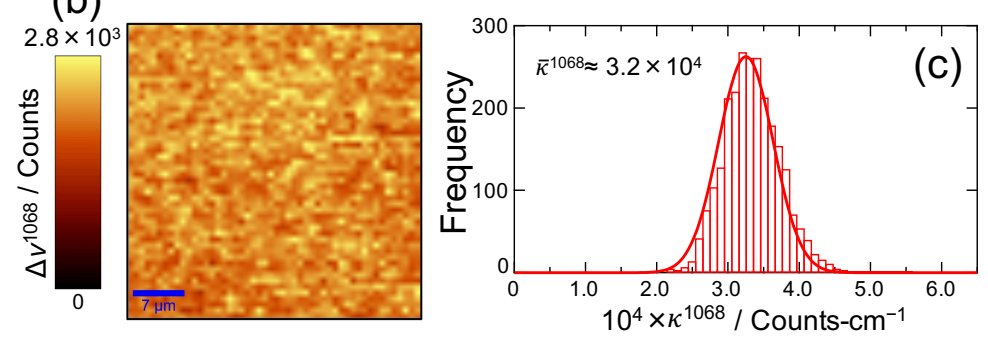

Figure S4. Analysis of SERS uniformity from nanostructured PC-Au surface $\left(\mathrm{t}_{\mathrm{Ti}}=0 \mathrm{~nm}\right)$. (a) Measured (blue trace) spectrum from surface with pitch $\Lambda_{\mathrm{g}}=250 \mathrm{~nm}$, nanogap $g \approx 10 \mathrm{~nm}$, and modeled Lorentzian bands (red trace). (b) Raman image of the integrated intensity of the $1068 \mathrm{~cm}^{-1}$ band over a $1600 \mu \mathrm{m}^{2}$ area. (c) Histogram of integrated intensity of $1068 \mathrm{~cm}^{-1}$ vibration mode over the scanned area $(n=2500)$. 
5. Au dielectric function. The excitation of the LSPR modes on metal nanostructures depends on the characteristics of the metal dielectric function $\varepsilon(\omega)=\varepsilon_{1}(\omega)+i \varepsilon_{2}(\omega)$, where $\varepsilon_{1}\left(\omega_{0}\right)<0$ is required to satisfy the resonance condition for a surrounding medium with dielectric constant $\varepsilon_{\mathrm{d}}>0$ at resonance frequency $\omega_{0}$ which also depends on the geometry of the nanostructure. The large negative $\varepsilon_{1}$ of $\mathrm{Au}$ result in LSPR modes with an enhanced electric field in the near-zone region of the nanostructure, which is an important property of nanoplasmonic resonators. However, $\varepsilon_{2}$ represents losses related to electron interactions, free-electron intraband transitions, and interband transitions within the conduction band that limits the resonance quality-factor and the electric field enhancement. The free-electron Drude-Sommerfeld (DS) dielectric function model is expressed as $\varepsilon(\omega)=1+\chi^{\mathrm{DS}}(\omega)$, where the susceptibility is $\chi^{\mathrm{DS}}(\omega)=\omega_{\mathrm{p}}{ }^{2} /\left(\omega^{2}+i \gamma \omega\right), \omega_{\mathrm{p}}$ is the bulk resonance frequency of the metal, and $\gamma$ is the intrinsic electron damping rate that accounts for the intrinsic energy loss of the resonator. The susceptibility can be expanded to $\chi^{\mathrm{DS}}(\omega) \approx \omega_{\mathrm{p}}{ }^{2} / \omega^{2}+i \omega_{\mathrm{p}}{ }^{2} \gamma / \omega^{3}$, assuming that $\omega>>\gamma$. Therefore, the components of the dielectric function are $\varepsilon_{1}(\omega) \approx \omega_{\mathrm{p}}{ }^{2} / \omega^{2}$ and $\varepsilon_{2}(\omega) \approx \omega_{\mathrm{p}}{ }^{2} \gamma / \omega^{3}$. For PC-Au, the DS model provides a good fit to the data for wavelengths above $600 \mathrm{~nm}$, but diverges for shorter wavelengths due to interband transitions, thus requiring an additional susceptibility term as $\varepsilon(\omega)=1+\chi^{\mathrm{DS}}(\omega)+\chi^{\mathrm{IB}}(\omega){ }^{4}$. The damping rate is the primary parameter for quantifying the LSPR quality-factor, which is estimated as $Q \equiv \omega / 2 \gamma \approx\left(\omega \partial \varepsilon_{1} / \partial \omega\right) / 2 \varepsilon_{2}$, regardless of the geometry of the nanostructure. ${ }^{5}$ The damping rate is inversely proportional to the Drude relaxation time $\gamma=1 / \tau_{\mathrm{D}}$, and for PC-Au $\tau_{\mathrm{D}} \approx 14 \pm 3$ fs for wavelengths above $500 \mathrm{~nm}$, which results in an intrinsic quality-factor of $Q \sim 20$, and is an upper limit for PC-Au nanoplasmonic resonators. ${ }^{6}$ In general, a small $\partial \varepsilon_{1} / \partial \omega$ and large $\varepsilon_{2}$ tend to tend to smear out the LSPR. For Ti, $\partial \varepsilon_{1} / \partial \omega$ is smaller by a factor of approximately $14 \times$ and $\varepsilon_{2}$ is greater by more than a factor of $10 \times$, compared to $\mathrm{Au}$, both of which contribute to strong LSPR damping by Ti. Plasmon damping by $\mathrm{Cr}$ is more severe due to the large values of $\varepsilon_{2}$.

6. 6a(a1) vibration band. The $6 \mathrm{a}(\mathrm{a} 1)$ vibration band at $695 \mathrm{~cm}^{-1}$ for $t_{\mathrm{Ti}}=3 \mathrm{~nm}$ has a much larger intensity ratio $I_{3} / I_{0}$ and drastically broadened vibration linewidth $\Delta_{3} \approx 43 \mathrm{~cm}^{-1}$ compared to the other vibration bands. And the emergence of the broad band near $712 \mathrm{~cm}^{-1}$ for $t_{\mathrm{Ti}}=5 \mathrm{~nm}$ with $I_{5} / I_{0}$ $\approx 0.73$ and $\Delta_{5} \approx 196 \mathrm{~cm}^{-1}$. The bands at $695 \mathrm{~cm}^{-1}$ and $712 \mathrm{~cm}^{-1}$ may be due to the $6 \mathrm{a}\left(\mathrm{a}_{1}\right)$ band of benzenethiol as well as enhanced Raman vibration bands of amorphous $\mathrm{TiO}_{2}$, which has been reported to be due to the overlap of the vibration band of the anatase and rutile phases of $\mathrm{TiO}_{2}$, at $639 \mathrm{~cm}^{-1}$ and $826 \mathrm{~cm}^{-1}$, respectively. ${ }^{18}$ As the concentration of Ti increases, the vibration band shifts near to the midpoint between the vibration bands of the anatase and rutile phases, and increased band intensity, as well as significant broadening, which has been previously reported due to the structural disorder of a deposited $\mathrm{TiO}_{2}$ thin film. ${ }^{19}$

7. Virtual bound states. The characteristics of surface plasmons are determined by the intrinsic electronic structure of the supporting metal. Metals have hybridized $s p$ bands that resemble freeelectron behavior, with a density of states that varies weakly near the Fermi energy. Figure S5 shows a schematic of the band structure for Au near the $X$ and $L$ symmetry points. ${ }^{7}$ The dominant contributions to the $d$-sp interband transition are shown, with onset near $\Delta_{\mathrm{X}} \approx 1.9 \mathrm{eV}$ and increase 
near $\Delta_{\mathrm{L}} \approx 2.4 \mathrm{eV} .{ }^{8}$ For photon energies below $\Delta_{\mathrm{X}}$, the optical response of the metal is determined by indirect intraband excitations. When transition metals are placed in a host metal, the impurity electrons can form virtual bound states with a density of states located near the Fermi energy $E_{\mathrm{f}}$ of the host metal. ${ }^{9,10}$ The number of electrons contained in the impurity state depends on its position relative to the host $E_{\mathrm{f} .}{ }^{12}$ For the AuTi alloy, the virtual bound state of the impurity is expected to lie above $E_{\mathrm{f}}$ and is assumed to have a Lorentzian band shape centered at energy $E_{\mathrm{d}}$ with a full-width half-maximum given by $\Delta_{\mathrm{d}}{ }^{11,12}$ Figure S5 shows a schematic of the total density of states of the Au host and the interdiffused Ti impurities. The virtual bound state affects optical absorption by modifying the free-electron relaxation time, and by broadening the interband transition bands of $\mathrm{Au}$ to lower energies. ${ }^{13}$ Based on the Friedel-Anderson model for degenerate non-magnetic virtual bound state alloys, the dielectric function of the host is modeled as $\varepsilon(\omega)=1+\chi^{\mathrm{DS} 2}(\omega)+\chi^{\mathrm{IB}}(\omega)+$ $\chi^{\mathrm{VBS}}(\omega)$, where $\chi^{\mathrm{DS} 2}$ and $\chi^{\mathrm{VBS}}$ are introduced to the dielectric function representing the change to the relaxation time and interband transitions, respectively, by the transition metal alloy. ${ }^{14-16}$ The first term is the DS-model generalized to allow for an energy dependent relaxation term due to freeelectron scattering by the impurity states. The modified DS model is expressed as $\chi^{\mathrm{DS} 2} \approx \omega_{\mathrm{p}}{ }^{2} / \omega^{2}+$ $i \omega_{\mathrm{p}}{ }^{2} / \omega^{3} \tau(\omega)$, where the first term is $\varepsilon_{1}(\omega)$ and is unchanged from the DS model and the second term is related to $\varepsilon_{2}(\omega) \approx \omega_{\mathrm{p}}{ }^{2} / \omega^{3} \tau(\omega)$, where $\omega_{\mathrm{p}}$ is the plasma frequency of the Au host and $\tau(\omega)$ is the total frequency dependent relaxation, which is expressed as $1 / \tau(\omega)=1 / \tau_{\mathrm{D}}+C / \tau_{i}(\omega)$, where $\tau_{i}(\omega)$ is relaxation time related to the impurity and $C$ is the fractional atomic impurity concentration. ${ }^{16,17}$ The impurity relaxation time is a function of $E_{d}$ and $\Delta_{\mathrm{d}}$ as $1 / \tau_{i}(\omega) \equiv \mathrm{f}\left(E_{d}, \Delta_{\mathrm{d}}\right) .{ }^{11,16}$ The second term represents impurity-induced interband absorption as $\chi^{\mathrm{VBS}}=c \hbar\left[\left(\omega_{\mathrm{d}}\right)^{2} / \omega\right] \pi\left\langle N_{\mathrm{d}}(E)\right\rangle$, where $\omega_{\mathrm{d}}$ is the matrix element for interband transitions and $N_{\mathrm{d}}$ is the impurity density of $d$-states that is assumed to have a Lorentzian shape. ${ }^{14,16}$ Therefore, the change in $\varepsilon_{2}$ after alloying is related to $1 / \tau_{i}(\omega)$ and the interband absorption of the impurity state.

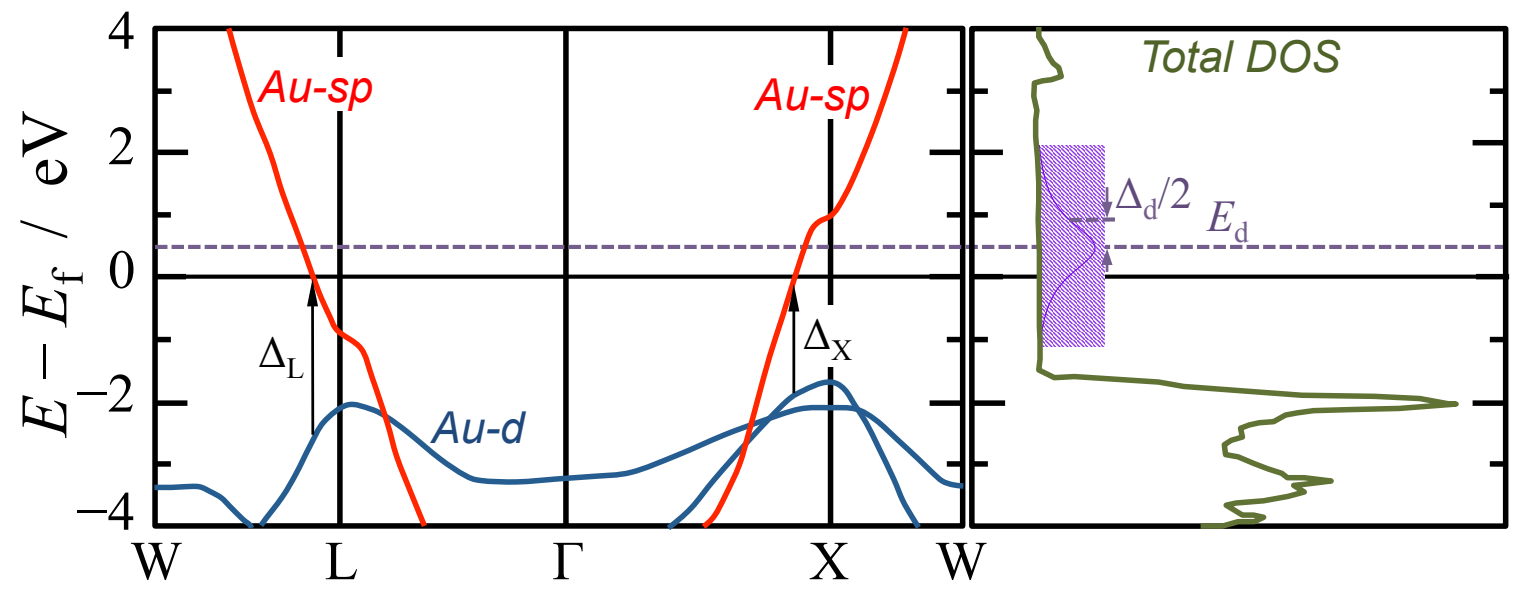

Figure S5. Diagrams of the electronic band structure and density of states of Au adapted from Ref. 7. The onset of interband transitions, from the occupied $d$-bands to the unoccupied $s p$-bands above the Fermi energy $E_{\mathrm{f}}$, occur near the $X$-symmetry point $\Delta_{\mathrm{X}} \approx 1.9 \mathrm{eV}$ and a sharp transition near the $L$-symmetry point $\Delta_{\mathrm{L}} \approx 2.4$ $\mathrm{eV}$. The virtual bound states of AuTi impurities are located above the Fermi energy centered at $E_{\mathrm{d} .}{ }^{11}$

The optical absorption properties of virtual bound states formed by several dilute transition metal alloys have been reported by Beaglehole. ${ }^{16}$ Tangamonsiri et al. studied the optical absorption properties of dilute $\mathrm{Ti}$ concentrations ranging up to 2.5 at.\% of $\mathrm{Ti}$ in a $\mathrm{Au}$ host. ${ }^{11}$ For low 
concentrations of $\mathrm{Ti}$, the optical absorption characteristics of the pure $\mathrm{Au}$ sample did not change significantly. As the amount of Ti impurity was increased, the strong absorption of pure Au, due the host interband transitions near $\Delta_{\mathrm{X}}$ and $\Delta_{\mathrm{L}}$ (Fig. S5), decreased and a broad absorption peak appeared at lower energies that extended as low as $1.6 \mathrm{eV}$. Based on the Friedel-Anderson virtual bound states model, $E_{\mathrm{d}} \approx(0.5 \pm 0.2) \mathrm{eV}$ above $E_{\mathrm{f}}$ with $\Delta_{\mathrm{d}} \approx(1.2 \pm 0.4) \mathrm{eV}$, as shown schematically in Fig. S5. ${ }^{11}$

7. Simulation models. 2D finite-difference time-domain (FDTD) calculations were performed with Fullwave (RSoft, Inc.). Periodic boundaries are used in the x-direction. Perfectly matched layer (PML) boundary conditions of $8 \mathrm{~nm}$ we used at the grid edges in the z-direction. A nonuniform grid with nominal $1 \mathrm{~nm}$ grid spacing was used. Complex frequency-dependent dielectric functions $\varepsilon(\omega)$ of $\mathrm{Au}$ and $\mathrm{Ti}$ are represented by the Brendel-Borman model that are included in the simulation code. Simulations at all incident frequencies were checked for convergence. A time step of $2 \times 10^{-4}$ $c T$ units $\left(c=2.99792458 \times 10^{8}\right)$ and simulation time of $30 c T$. Figure S6(a) shows details of the model and Fig. S6(b) shows an example of the field profile $\left|\boldsymbol{E} / \boldsymbol{E}_{\mathrm{i}}\right|$ generated in the nanocavity with TM incident radiation at the resonance frequency.

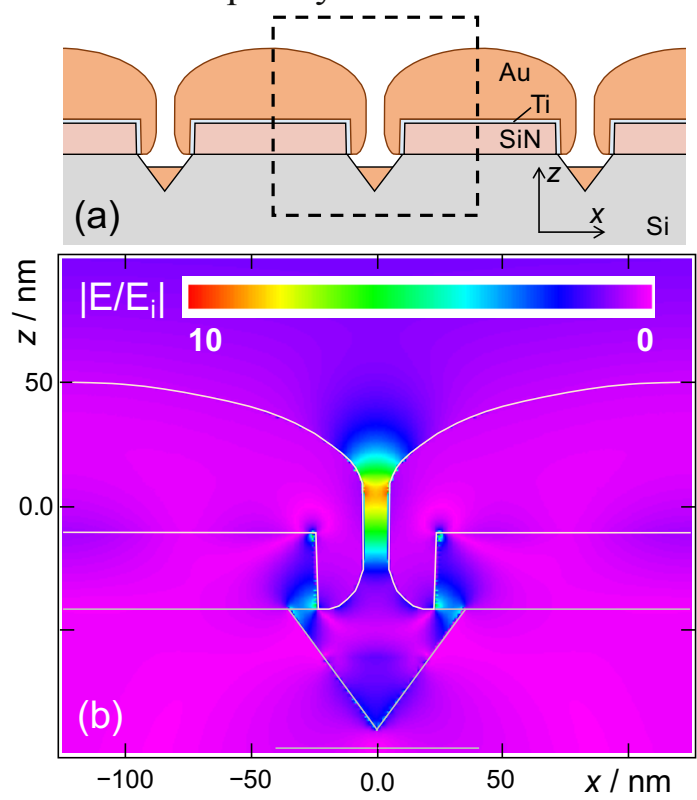

Figure S6. 2D FDTD model with pitch $\Lambda_{\mathrm{g}}=250 \mathrm{~nm}$ and nanogap spacing $g=10 \mathrm{~nm}$. (a) Simulation region in black dashed region. Periodic boundary condition in $x$-direction. PML boundary conditions in $z$-direction.

(b) Electric field $\left|\boldsymbol{E} / \boldsymbol{E}_{\mathrm{i}}\right|$ generated in the nanocavity with TM incident radiation at the resonance frequency.

\section{References}

1. Tisone, T. C.; Drobek, J. Diffusion in Thin-Film Ti-Au, Ti-Pd, and Ti-Pt Couples. J. Vac. Sci. Technol. 1972, 9, 271-275.

2. Martinez, W. E.; Gregori, G.; Mates, T. Titanium Diffusion in Gold Thin Films. Thin Solid Films 2010, 518, 2585-2591.

3. Habteyes, T. G.; Dhuey, S.; Wood, E.; Gargas, D.; Cabrini, S.; Schuck, P. J.; Alivisatos, A. P.; Leone, S. R. Metallic Adhesion Layer Induced Plasmon Damping and Molecular Linker as a Nondamping Alternative. ACS Nano 2012, 6, 5702-5709.

4. Kreibig, U.; Vollmer, M. Optical Properties of Metal Clusters; Springer-Verlag: Berlin, 1995. 
5. Wang, F.; Shen, Y. R. General Properties of Local Plasmons in Metal Nanostructures. Phys. Rev. Lett. 2006, 97, 206806.

6. Olmon, R. L.; Slovick, B.; Johnson, T. W.; Shelton, D.; Oh, S.-H.; Boreman, G. D.; Raschke, M. B. Optical Dielectric Function of Gold. Phys. Rev. B 2012, 86, 235147.

7. Ladstädter, F.; Hohenester, U.; Puschnig, P.; Ambrosch-Draxl, C. First-Principles Calculation of Hot-Electron Scattering in Metals. Phys. Rev. B 2004, 70, 235125.

8. Le Thi Ngoc, L.; Wiedemair, J.; van den Berg, A.; Carlen, E. T. Plasmon-Modulated Photoluminescence from Gold Nanostructures and its Dependence on Plasmon Resonance, Excitation Energy, and Band Structure. Opt. Express 2015, 23, 5547-5564.

9. Anderson, P. W. Localized Magnetic States in Metals. Phys. Rev. 1961, 124, 41-53.

10. Abeles, F. Optical Properties of Au-Ni Alloys. J. de Physique et le Radium 1962, 23, 677-685.

11. Tangamonsiri, S.; Gilberd, P. W.; Kaiser , A. B. Virtual Bound States in AuTi Alloys. Solid State Commun. 1977, 24, 125-127.

12. Beaglehole, D. A Study of the Virtual Bound State Dielectric Constant. J. Phys. F: Met. Phys. 1975, 5, 657-668.

13. Kaiser, A. B.; Gliberd, P. W. Impurity Density of States of Dilute CuNi Alloys. J. Phys. F: Met. Phys. 1976, 6, L209-L214.

14. Caroli, B. Absorption Infra-Rouge Dans les Alliages Dilués Au Ni. Phys. Kondens. Mater. 1963, 1, 346-358.

15. Kjollerstrom, B. Calculation of the Dielectric Constant for a Dilute Magnetic Alloy. Phil. Mag. 1969, 19, 1207-1216.

16. Beaglehole, D. Optical Absorption and $s$ and $p$ Shifts of Virtual-Bound-State Alloys. Phys. Rev. B 1976, 14, 341-346.

17. Bassett, M.; Beaglehole, D. Optical Studies of Dilute AuNi Alloys. J. Phys. F: Met. Phys. 1976, 6, 1211-1221.

18. Degioanni, S.; Jurdyc, A.-M.; Bessueille, F.; Coulm, J.; Champagnon, B.; Vouagner, D. Surface-Enhanced Raman Scattering of Amorphous $\mathrm{TiO}_{2}$ Thin Films by Gold Nanostructures: Revealing First Layer Effect with Thickness Variation. Appl. Phys. Lett. 2013, 114, 234307.

19. Gouadec, G.; Colomban, P. Raman Spectroscopy of Nanomaterials: How Spectra Relate to Disorder, Particle Size and Mechanical Properties. Prog. Cryst. Growth Charact. Mater. 2007, 53, 1-56. 